\title{
ASYMPTOTICAL MODELS OF BOUNDARY LAYER FLOW CONTROL
}

\author{
I. I. Lipatov \\ Central Aerohydrodynamic Institute (TsAGI) \\ Zhukovsky Str. 1, Zhukovsky, Moscow Region, Russia
}

\begin{abstract}
Investigated are the local flows in the laminar boundary layers in the vicinity of heating elements. On the basis of asymptotical analysis, mathematical models are formulated and similarity parameters are found. Determined are the flow parameters providing flow control (separation, transition). Presented are the results of numerical and analytical analysis. Another method of the flow control is associated with the technology of new materials development. For example, porous metals allow use of passive control methods to influence boundary layer separation or laminar-turbulent transition.
\end{abstract}

\section{LOCAL SURFACE HEATING}

Among different methods of boundary-layer flow control, one of the mostly investigated now is a method associated with energy release due to electrical discharge, surface heating, or cooling.

The aim of this paper is the analysis of possible application of the local surface heating to determine response of the boundary-layer flow and to find optimal heating elements parameters.

It is supposed that on the surface of the body, there are located heated parts, having temperatures different from the gas temperature in ambient boundary layer flow. Also, it is supposed that temperature difference may change with time. Practically, this method is easy to fulfill using electrically conducting strips. An example of such method application is described in [1].

The most important factor due to energy release (surface heating) is density change in the region influenced by the heating. This region structure is controlled by the convection and thermal conductivity processes. At the same time, density change (diminishing due to temperature rise) will change boundary-layer thickness. Situation is similar to the local flow nearby local surface distortion, but in the present case, effective surface distortion is created due to temperature

This is an Open Access article distributed under the terms of the Creative Commons Attribution-Noncommercial License 3.0, which permits unrestricted use, distribution, and reproduction in any noncommercial medium, provided the original work is properly cited. 
(density) change. The difference is that the distortion shape is not known beforehand but is formed due to energy release in the boundary layer and due to the region with smaller density formation. Previous analysis of the disturbed flow nearby local surface distortion allowed to develop corresponding mathematical problems and to find distortion parameters influencing boundary layer flow [2].

In the paper, the corresponding asymptotical analysis is applied to analyze energy release influence on the separation of the boundary layer flow along with the influence on the boundary layer flow instability.

Investigated are the local flows in the laminar boundary layer nearby heating elements located on the surface and flows near porous surface. On the basis of asymptotic analysis, mathematical models are derived and similarity parameters are found. Described are unsteady local heating regimes providing boundarylayer separation and laminar-turbulent transition control. Presented are numerical and analytical results.

At the present time, intensive investigations are conducted associated with new methods of boundary-layer control due to energy release. The energy source can be due to the electrical discharge, surface heating or cooling. Along with these methods, mechanical devices are analyzed, so-called MEMS (microelectromechanical systems), or methods associated with suction or injection. Application of such methods allows suppressing boundary-layer separation to change boundary-layer transition position along with the influence on the turbulent boundary-layer parameters.

This paper is aimed at the analysis of possible regimes of local surface heating to determine arising disturbances in the boundary layers. It is supposed as well to formulate corresponding mathematical problems and to determine similarity parameters. Analogous analysis is done for disturbed flows near porous surface.

It is supposed that on the streamlined surface, there are located parts having temperature which differs from the temperature of an ambient gas. It is supposed as well that this temperature depends on time. Practically, it is easy to fulfill such method of control having conducted wires or strips and using electrical current for local heating [1].

Let us consider physical aspects of such flows. The most important factor associated with the surface heating is gas density change in the region where energy release influence is significant. Dimensions of this region are determined by convection and heat conduction processes. Temperature increase will lead to the density decrease which will change boundary-layer thickness. This change may induce in the external inviscid flow corresponding pressure disturbances. Situation is very similar to the flow in the boundary layer with the surface distortions. The main difference is that, in the considered case, geometry of surface distortions is not known beforehand but is determined by energy release and by corresponding decreased density region formation. Flow analysis in the boundary layer disturbed by an abrupt change of surface temperature and catalytic properties distribution is presented in [3-5]. 
For the subsequent analysis, the results of $[4,6-8]$ were used in which local surface distortions located on the bottom of the surface were analyzed. It is supposed that the Reynolds number is large but does not exceed the critical value corresponding to the laminar-turbulent transition. Subsequent analysis is based on the derivation of estimates of possible physical mechanisms and similarity parameters determination.

Considered is the supersonic or subsonic viscous gas flow near a flat semiinfinite plate. It is supposed that the Reynolds number is large but is subcritical corresponding to the laminar flow

$$
\operatorname{Re}=\frac{\rho_{\infty} u_{\infty} l}{\mu_{\infty}}=\varepsilon^{-2} \rightarrow \infty
$$

where $\rho_{\infty}, u_{\infty}$, and $\mu_{\infty}$ are the density, longitudinal velocity, and dynamical viscosity coefficient in undisturbed flow over the region where heated part of plate is located; and $l$ is the distance from a leading edge to a zone of energy release. The next nondimensional values are chosen for the Cartesian coordinates, velocity vector components, density, pressure, and dynamical viscosity coefficient: $x l$, $y l, z l, l u_{\infty}^{-1} t, u_{\infty} u, u_{\infty} v, u_{\infty} w, \rho_{\infty} \rho, u_{\infty}^{2} R^{-1} T, \mu_{\infty} \mu$.

In general case, it is supposed that temperature change in the local region is finite $\Delta T \sim T \sim O(1)$, and the region of increased temperature is characterized by the longitudinal size $a \leq O(1)$, transversal size $b \leq O(1)$, and characteristic time of temperature change $O(\tau)$.

Undisturbed flow is two-dimensional (2D) and steady but disturbed flow is supposed to be unsteady and three-dimensional (3D). Preliminary analysis is conducted for $2 \mathrm{D}$ flow and then results are generalized for 3D flows.

Following the method of matched asymptotic expansions [7], at the beginning, let consider the region having identical sizes comparable with the body length: $x \sim y \sim z \sim O(1)$. For large Reynolds number, the flow in this region is described by the Euler equations. For the flat plat having zero angle of attack and zero thickness, these equations solution is a solution describing undisturbed flow. To fulfill no-slip conditions, it is necessary to introduce boundary layer region located nearby the surface and having the following sizes: $x \sim z \sim O(1)$ and $y \sim O(\varepsilon)$.

Local surface heating can lead to the effective distortion formation, thickness of which can be evaluated using longitudinal impulse equation.

Surface temperature change will cause corresponding gas density change in the layer located nearby the surface $\Delta \rho \sim \rho \sim O(1)$. If the Prandtl number is finite: $\operatorname{Pr} \sim O(1)$ in general case, it may be deduced that thickness of local viscous layer and thickness of temperature conducting layer are comparable. In the near-wall layer, longitudinal velocity is proportional to the distance from the wall $y / \varepsilon: u \sim O(y / \varepsilon)$. If values of convective and diffusion terms in the longitudinal momentum equation have the same order, then the next estimate 
can be obtained for the thickness of local layer as a function of its longitudinal size $a$ :

$$
y \sim O\left(\varepsilon \alpha^{1 / 3}\right) \leq O(\varepsilon)
$$

Subsequent analysis depends on the longitudinal size of heated part of the surface. At least three different regimes described by different mathematical models may be formulated.

The first one corresponds to the longitudinal size smaller than the boundary layer thickness. If the sizes of the disturbed region have the same order:

$$
\alpha \sim O\left(\varepsilon \alpha^{1 / 3}\right), \alpha \sim \varepsilon^{3 / 2},
$$

one will get the disturbed region where the flow is described by Navier-Stokes equations with boundary conditions taking into account rarefied gas effects (slip conditions). Characteristic time in this region has the following order: $\tau \sim \varepsilon^{-1}$.

For relatively larger sizes of the heated part $\alpha^{2} \operatorname{Re}^{3 / 2}=\operatorname{Re}_{1} \rightarrow \infty$, the disturbed flow will be described by so-called equations for compensation regime [8]. This regime will exist for length comparable with the boundary-layer thickness as well as for larger sizes but lesser than the length scale comparable with the so-called free interaction scale. Corresponding to this regime,

$$
a \sim O\left(\varepsilon^{3 / 4}\right) ; \Delta y \sim O\left(\varepsilon^{5 / 4} \Delta T\right) ; \Delta p \sim O\left(\varepsilon^{1 / 2} \Delta T\right) .
$$

The corresponding mathematical problem can be written as follows

$$
\begin{gathered}
\mathrm{Sh}_{2} \frac{\partial u_{b}}{\partial t_{b}}+u_{b} \frac{\partial u_{b}}{\partial x_{b}}+v_{b} \frac{\partial u_{b}}{\partial y_{b}}+\Pi_{1} w_{b} \frac{\partial u_{b}}{\partial z_{b}}+T_{b} \frac{\partial p_{b}}{\partial x_{b}}=\frac{\partial^{2} u_{b}}{\partial y_{b}^{2}} \\
\mathrm{Sh}_{2} \frac{\partial w_{b}}{\partial t_{b}}+u_{b} \frac{\partial w_{b}}{\partial x_{b}}+v_{b} \frac{\partial w_{b}}{\partial y_{b}}+\Pi_{1} w_{b} \frac{\partial w_{b}}{\partial z_{b}}+T_{b} \frac{\partial p_{b}}{\partial z_{b}}=\frac{\partial^{2} w_{b}}{\partial y_{b}^{2}} \\
\operatorname{Sh}_{2} \frac{\partial T_{b}}{\partial t_{b}}+u_{b} \frac{\partial T_{b}}{\partial x_{b}}+v_{b} \frac{\partial T_{b}}{\partial y_{b}}+\Pi_{1} w_{b} \frac{\partial T_{b}}{\partial z_{b}}=\frac{\partial^{2} T_{b}}{\partial y_{b}^{2}} \\
\frac{\partial u_{b}}{\partial x_{b}}+\frac{\partial v_{b}}{\partial y_{b}}+\frac{\partial w_{b}}{\partial z_{b}}=0 ; \\
u_{b}\left(x_{b}, 0, z_{b}, t_{b}\right)=w_{b}\left(x_{b}, 0, z_{b}, t_{b}\right)=v_{b}\left(x_{b}, 0, z_{b}, t_{b}\right)=0 ; \\
T_{b}\left(x_{b}, 0, z_{b}, t_{b}\right)=T_{w}\left(x_{b}, z_{b}, t_{b}\right) ; \\
u_{b} \rightarrow y_{b}+d ; \quad d_{1}=\int_{0}^{\infty}\left(1-T_{1}\right) d \eta+d \\
T_{1}(x, \infty) \rightarrow 1 ; y_{b} \rightarrow \infty ; \\
d(-\infty) \rightarrow 0 ; \quad P_{b}\left(x_{b}, t\right)=-B_{2} \frac{\partial d_{1}}{\partial x_{b}} .
\end{gathered}
$$




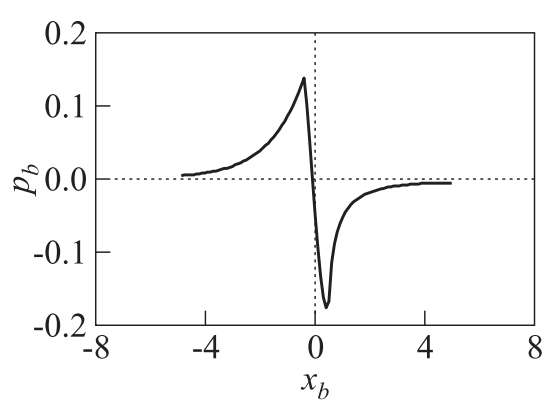

Figure 1 Induced pressure distribution

This problem was numerically solved. Numerical results were obtained for the following surface temperature distribution

$$
\begin{gathered}
T_{w}\left(\left|x_{b}\right| \leq 0.5, t\right)=1+(1-\exp (-t))\left(0.25-x_{b}^{2}\right) ; \\
T_{w}\left(\left|x_{b}\right|>0.5, t\right)=1 ; \quad B_{2}=1 .
\end{gathered}
$$

In Fig. 1, induced pressure distribution is presented: $p_{b}\left(x_{b}, t \rightarrow \infty\right)$ for large time values.

\section{FLOWS NEAR POROUS WALLS}

Porous wall structure means that due to pressure difference on the external and internal sides of porous surface can lead to the distributed suction (in the regions of relatively high pressure) or distributed injection. In many cases, it can be supposed that mass transfer obeys the Darsy law (or linear dependence between vertical velocity on the wall and disturbed pressure distribution).

This boundary condition allows, in fact, to reconsider early obtained results [9-11] describing self-induced boundary-layer separation for the case of passive flow control.

Using results obtained in [9], mathematical problem for flows near porous walls can be formulated as follows:

$$
\begin{gathered}
u \frac{\partial u}{\partial x}+v \frac{\partial u}{\partial y}+\frac{\partial p}{\partial x}=\frac{\partial^{2} u}{\partial y^{2}} ; \quad \frac{\partial u}{\partial x}+\frac{\partial v}{\partial y}=0 ; \\
y=0: \quad v=-\beta p, \quad u=0 ; \\
y \rightarrow \infty: \quad \begin{aligned}
u & =y+A(x) ; \\
x \rightarrow-\infty &
\end{aligned} \\
p=-\frac{\partial A}{\partial x}
\end{gathered}
$$


This problem differs from the problems describing disturbed flow near impermeable wall due to condition for the vertical velocity on the wall [9-11]. In fact, such conditions are well known in fluid mechanics.

For small values of self-induced pressure, the following form of solution can be considered:

$$
u=y+u_{1} ; \quad v=v_{1} ; \quad p=p_{1} .
$$

This form of solution gives the following form of equations for the first approximation:

$$
\begin{gathered}
y \frac{\partial u_{1}}{\partial x}+v_{1}+\frac{\partial p_{1}}{\partial x}=\frac{\partial^{2} u_{1}}{\partial y^{2}} \\
\frac{\partial u_{1}}{\partial x}+\frac{\partial v_{1}}{\partial y}=0 .
\end{gathered}
$$

As usual, the solution can be presented in the normal mode approximation:

$$
\begin{gathered}
\left(u_{1}, v_{1}, p_{1}, A\right)=e^{a x}(U, V, P, B) ; \\
y \alpha U+V+\alpha P=U^{\prime \prime} \\
U(\infty)=B ; \quad y \alpha U^{\prime}=U^{\prime \prime \prime} .
\end{gathered}
$$

After some transformations, an Airy equation can be obtained:

$$
y=\alpha^{-1 / 3} Y ; \quad U^{\prime}=F ; \quad Y F=F^{\prime \prime}
$$

with the following solution:

$$
\begin{gathered}
F=C A i(Y) ; \quad F=\frac{\partial U}{\partial y}=\alpha^{1 / 3} \frac{\partial U}{\partial Y} ; \quad F=\frac{\partial U}{\partial Y}=C \alpha^{-1 / 3} A i(Y) \\
U(\infty)=\int_{0}^{\infty} F d Y=C \alpha^{-1 / 3} \int_{0}^{\infty} A i(Y) d Y=\frac{C}{3 \alpha^{1 / 3}} ; \quad P=-\alpha B=-\frac{C \alpha^{2 / 3}}{3} \\
(\alpha-\beta) P=\alpha^{2 / 3} U_{w}^{\prime \prime}=C \alpha^{1 / 3} A i^{\prime}(0) ; \\
-\frac{C \alpha^{2 / 3}}{3}(\alpha-\beta)=C \alpha^{1 / 3} A i^{\prime}(0) .
\end{gathered}
$$

Ley introduce new variables:

$$
\alpha^{1 / 3}(\alpha-\beta)=-3 A i^{\prime}(0) ; \quad \alpha=\delta^{3} \beta .
$$

Eventually, one obtains the following relation associating increment of growth and wall velocity parameter $\beta$ :

$$
\delta\left(\delta^{3}-1\right)=-\frac{3 A i^{\prime}(0)}{\beta^{4 / 3}} .
$$


The following two limits can be considered.

The first one corresponding to small porosity $\beta \rightarrow 0$ :

$$
\delta \approx\left[-\frac{3 A i^{\prime}(0)}{\beta^{4 / 3}}\right]^{1 / 4} \approx \frac{1}{\beta^{1 / 3}}\left[-3 A i^{\prime}(0)\right]^{1 / 4} .
$$

Then, since $\alpha=\delta^{3} \beta$, one gets:

$$
\alpha \approx\left[-3 A i^{\prime}(0)\right]^{3 / 4}
$$

The same result as in classical case (impermeable plate) [9] is obtained.

The second limit corresponds to large porosity $\beta \rightarrow \infty$ :

$$
\delta \approx 1, \quad \alpha \approx \beta
$$

Large $\alpha$ values correspond to small length of disturbed region.

It is important that new boundary condition on the wall is linear in the approximation considered; so, mathematical problem for linear regimes can be considered as a uniform one.

To investigate stability problems, it is needed to consider unsteady mass transfer regimes.

\section{REFERENCES}

1. Yurchenko, N., G. Voropaev, R. Pavlovsky, P. Vinogradsky, and A. Zhdanov. 2003. Flow control using variable temperature boundary conditions. Proc. European Fluid Mecahics Conference EFMC-2003. Toulouse, France.

2. Neyland, V. Ya., V. V. Bogolerov, G. N. Dudin, I. I. Lipatov, et al. 2007. Asymptotical problems of the supersonic viscous gas flows. Elsevier. $456 \mathrm{p}$.

3. Sokolov, L. A. 1975. To the asymptotic theory of 2-D laminar boundary layer flows with the abrupt change of surface temperature. Tr. TsAGI 1650:18-23.

4. Gershbein, E. A., V. Yu. Kazakov, and G. A. Tirsky. 1986. Laminar boundary layer development downstream from the point of abrupt change of surface catalytic properties. TVT 24(6).

5. Bogolepov, V.V., I. I. Lipatov, and L. A. Sokolov. 1990. Structure of chemically nonequilibrium flows in the vicinity of an abrupt change of temperature and catalytic properties of the surface. PMTF 3:30-41.

6. Bogolepov, V. V., and V. Ya. Neyland. 1971. Flow near local surface distortions in the external supersonic flow. Tr. TsAGI 1363.

7. Van Dyke, M. 1967. Methods of disturbancies in fluid mechanics. Moscow: Mir. $310 \mathrm{p}$.

8. Bogolepov, V. V., and V. Ya. Neyland 1976. Investigation of locally disturbed viscous supersonic flows. In: Aeromekhanika. Moscow: Nauka. 104-18. 
9. Neyland, V. Ya. 1969. To the theory of laminar boundary layer separation in supersonic flow. Izv. AN SSSR, MZhG. 4:53-57.

10. Stewartson, K. 1969. On the flow near the trailing edge of a flat plate. Mathematika 16(1(31)):106-21.

11. Messiter, A.F. 1970. Boundary layer near the trailing edge of a flat plate. SIAM J. Appl. Math. 18(1):241-57. 